\title{
REVISIÓN/REVIEW PAPER
}

Recibido: 15/07/2015---Aceptado: 30/10/2015---Publicado: 15/12/2015

\section{LA HERMENÉUTICA COMO ANCLAJE EPISTÉMICO DEL MÉTODO ETNOGRÁFICO}

Johnny Alarcón Puentes'‥Universidad del Zulia. Venezuela jalarconxxi@gmail.com

\section{RESUMEN}

En este trabajo se abordan elementos que permiten establecer una relación entre la práctica antropológica y la hermenéutica. Partimos desde los clásicos de la teoría hermenéutica para constituir unos criterios relevantes que permitan analizar la realidad cultura. Rompemos con los paradigmas estáticos que no permiten una postura creativa a la hora de examinar los datos etnográficos. Construimos una perspectiva hermenéutica desde el quehacer antropológico, para luego definir su campo de acción a partir del método etnográfico. Concluimos, que desde un nuevo enfoque la antropología debe salir de los ámbitos empirista del positivismo para lograr una configuración de los contextos culturales.

Palabras clave: Método etnográfico - Hermenéutica - Episteme - Cultura

\section{HERMENEUTICS AS EPISTEMOLOGICAL ANCHORING OF THE ETHNOGRAPHIC METHOD}

\begin{abstract}
This paper addresses elements that allow us to establish a relationship between the anthropological practice and hermeneutics. We start from the classics of the hermeneutical theory to establish relevant criteria that permits to analyze the culture reality. We break with the static paradigms which do not allow a creative stance in considering the ethnographic data. We build a hermeneutical perspective from the anthropological work, and then define its field of action from the ethnographic method. We conclude that, from a new focus, Anthropology should leave empiricist areas of positivism to reach the configuration of cultural contexts.
\end{abstract}

\footnotetext{
${ }^{1}$ Johnny Alarcón Puentes: Licenciado en Historia, Magister en Historia de Venezuela y en Antropología. Doctor en Ciencias Humanas y en Antropología. Profesor Titular de la Universidad del Zulia. Ponente en más de cuarenta congresos nacionales e internacionales. Autor de más de cuarenta publicaciones entre libros y artículos en revistas científicas. Investigador responsable de más de una decena de Proyectos de Investigación financiados por organismos nacionales.
} 
Key words: Ethnographic method - Hermeneutics - Episteme - Culture

\section{INTRODUCCIÓN}

En la actualidad la antropología se encuentra redefiniendo sus postulados teóricos a partir de novedosas posturas epistémicas. En la siguiente investigación se traza una perspectiva de análisis de la antropología teniendo como sustento la hermenéutica. En este sentido, la hermenéutica representa para la antropología el anclaje epistémico necesario para renovarse. Partimos de las concepciones tradicionales de la hermenéutica para, poco a poco, adentrarnos en las definiciones y análisis más contemporáneos que nos darán luces para reinterpretar el andamiaje teórico.

Para reconstituir, con criterios teóricos, que superen la visión descriptiva de la antropología tradicional, debe pasar por una revisión seria y un re/planteo riguroso de su epistemología, es decir, el rescate hermenéutico como esencia científica. Con esto intentamos superar la rigidez y unilateralidad refutando la concepción de objetividad del positivismo como acción externa a los individuos. Por ello planteamos la recuperación del contexto social, sus significados, representaciones y múltiples determinaciones.

Lo esencial en el análisis antropológico es el sujeto y la subjetividad como creadores y constructores de la realidad. Con el rescate hermenéutico para la antropología nos apropiamos de la perspectiva comprensiva/interpretativa para analizar los datos atendiendo las múltiples subjetividades presentes en el contexto social para de esta manera visualizar las diversas vertientes de la cotidianidad observada. La hermenéutica retoma así su papel dentro de la antropología como fundamento epistémico. De esta manera, desde el punto de vista metódico, la interpretación de la realidad y de los significados subjetivos de la acción social se transfigura en parte del hecho etnográfico de análisis. Con este nuevo enfoque la etnografía adquiere relevancia la interpretación en el estudio de los fenómenos culturales.

La etnografía no puede quedarse en una perspectiva reduccionista/descriptiva de lo cultural, no es solo acumulación de datos. Por el contario, la etnografía se despliega en un conjunto de operaciones para ir al campo y para luego construir teóricamente un texto escrito.

Utilizar la hermenéutica en la etnografía nos permite, una compresión/interpretación de los procesos culturales que sería imposible con una mera descripción social

Este trabajo lo dividimos en dos partes. La primera que aborda todo lo relativo a la hermenéutica y su entrelazamiento con la antropología y la segunda que versa sobre la definición de la etnografía como método que se despliega desde la hermenéutica.

\section{LA HERMENÉUTICA COMO FUNDAMENTO EPISTÉMICO DE LA ANTROPOLOGÍA}


Para esta investigación es importante abordar la realidad desde en el paradigma interpretativo que hunde sus raíces en la hermenéutica que, en todo caso, es considerado por todos los teóricos como un modelo distinto del positivismo. A pesar que la antropología en sus inicios es sólidamente positivista, creemos que ha logrado vencer los obstáculos que la ataban a ese paradigma para avanzar en diferentes direcciones, proporcionándonos un arsenal teórico múltiple que nos permiten acometer la empresa de la diversidad.

La hermenéutica representa para las ciencias sociales toda una apertura teórico/metódica que permite la comprensión (Verstehen) de los fenómenos sociales a la luz de elementos concretos y empíricos.

El concepto de hermenéutica viene del griego hermeneúcin, que significa el "arte de interpretar". Las primeras formas de aplicación de la hermenéutica la hicieron los griegos de la mano de la filología, es decir, la interpretación y comprensión de textos (Martínez, 2004: 102-103). Desde la teología cristiana se utiliza la hermenéutica como una forma de interpretar la biblia para penetrar los intersticios de la superficie del texto y así develar su significado. De la comprensión de la biblia, la hermenéutica es tomada por la ciencia jurídica para comprender textos dados y luego interpretar el sentido del basamento jurídico, a pesar que posteriormente se transforme en dogmático (Gutiérrez, 1986: 6-7).

Es con Friedrich Schleirmacher (1768-1834), cuando la hermenéutica tiene un sentido filosófico, pues para él la tarea de la hermenéutica era "entender el discurso tan bien como el autor, y después mejor que él". Intentó presentar una teoría coherente sobre el proceso de interpretación de los textos. Por ello es considerado el padre de la hermenéutica moderna (Gutiérrez, 1986: 13 y 14).

Schleirmacher propone un sistema circular que conocemos como el "círculo hermenéutico" que pretendía ser universal. Cada intérprete necesita introducirse en la dimensión social y la dimensión individual del autor para comprenderlo. En la medida en que el lector se identifique con el autor y se ponga en su lugar, tanto mejor será la interpretación (Martínez, 2004: 103-104).

WilhelmDilthey (1833-1911) comenzó el estudio de la hermenéutica inspirado por los trabajos de Schleirmacher. Ambos forman parte del movimiento romántico alemán. Dilthey puede ser considerado como un tipo empirista. La escuela hermenéutica inspirada por el romanticismo alemán siempre puso mucho énfasis en que el intérprete puede emplear su capacidad de comprensión y penetración en combinación con el contexto cultural e histórico del texto abordado para así obtener el sentido original del texto. Wilhelm Dilthey jamás dejó de aspirar a la posibilidad de una interpretación objetiva y universalmente válida de los textos. Dilthey aplicó el nombre que Friedrich Schleirmacher había dado al proceso de investigación hermenéutica que había fundado y también llamó a dicho proceso círculo hermenéutico. Este método fue considerado por Dilthey crucial para aportar el fundamento necesario a las "Geisteswissenschaften", "ciencias del espíritu". Que el proceso sea circular hace referencia a la interdependencia (circular y no inmediata) 
de significado entre el todo y sus partes. Claro está Dilthey fue un paso adelante de Schleirmacher al concebir una epistemología autónoma para la hermenéutica, además, concibió la hermenéutica como el estudio más allá de los textos escritos, es decir, la vida social misma y los múltiples significados que de ella emanan. Quizás esta visión se acerca un poco al trabajo antropológico.

Max Weber (1864-1920), al igual que Durkheim, intenta por todos los medios darle un status científico a las Ciencias Sociales. Para él, ese sitial lo establece la comprensión de los sistemas sociales a través de su herramienta heurística que denomino modelos ideales.

Esto es lo que Weber (2006) plantea sobre la comprensión:

Al igual que todo acaecer, la conducta humana ("externa" o "interna") muestra nexos y regularidades. Sin embargo, hay algo que es propio solamente de la cultura humana, al menos en sentido pleno: el curso de regularidades y nexos es interpretable por vía de la comprensión. Una "comprensión" de la conducta humana obtenida por medio de interpretación contiene ante todo una "evidencia" cualitativa específica, de dimensión singularísima (p. 175)

Sus enseñanzas abren una perspectiva metódica que arremete contra la pasividad, objetividad y la descripción del positivismo emparentada con la interpretación naturalista de lo social. La comprensión como elemento de análisis primordial permite articular los distintos hechos empíricos y verlos en una dimensión diferente, es decir, busca un equilibrio entre los procedimientos interpretativos y los observativos experimentales (Mardones, 1991: 251-254).

La inquietud de Weber por la naturaleza del capitalismo lo llevo al estudio de las instituciones sociales y culturales de occidente y compararla con oriente, en este sentido, el aporte a la antropología es vasto ya que hay toda una bibliografía referente a los aspectos socioculturales que no puede ser pasada por alto por los antropólogos. Sus estudios de lo económico amalgamado con otros elementos como la religión lo conducen a un enfoque metódico de definición de la sociedad como un sistema social vía única para la comprensión de la totalidad cultural. Pero independientemente, que exponga un criterio de totalidad deja muy claro que es imposible abarcar la realidad total y por ello el investigador debe hacer uso de abstracciones de los fenómenos y relaciones que se quiere estudiar. De acuerdo con esto, se niega a generalizar y universalizar las proposiciones de las ciencias de la cultura porque eso las haría intranscendentes y oscurecerían las diferencias significativas y necesarias. La afirmación anterior nos introduce en el mundo de la antropología contemporánea donde la diversidad y las diferencias de las culturas es lo primordial en los estudios de los complejos societarios. Es el quid de la antropología. 
Weber establece sus estudios partiendo de los modelos ideales, que no es más que la construcción de categorías, conceptos que se comportan como las características y las tendencias esenciales de los fenómenos de estudio, es decir, abstracciones que le permitían aprehender tanto hechos como valores. No pretendía por ninguna circunstancia forzar los datos para que se ajustaran al modelo, por el contrario, dice que el esquema teórico no debe ser confundido con la realidad. La comprensión de lo real equivale a la captación interpretativa del sentido con relación al tipo ideal construido, pues es sólo la experiencia quien va a determinar si la conducta real concuerda con lo construido. Estos tipos ideales podrían conducir a la ensoñación de prescribir leyes sociales, con lo cual Weber no estaba de acuerdo por creer que sólo se puede llegar explicitar probabilidades que puedan ser confirmadas por la observación. (Weber, 2006: 175-221). Por tanto, para Weber los tipos ideales son su herramienta heurística

En Weber (2006) podemos verificar una crítica concienzuda a los postulados de objetividad, sólo puede existir una explicación parcial, limitada y unilateral de los procesos sociales es imposible acceder a la verdad absoluta y definitiva. Pero esto no quiere decir que sólo exista en las investigaciones un parámetro de subjetividad, ya que con la comprensión de la realidad le estamos dando su carácter objetivo. (Pp. 222-269).

Weber (1984) explicando la sociología nos da su perspectiva de la subjetividad:

La palabra "sociología" se usa en muchos sentidos diversos. En el que aquí se adopta, sociología designa la ciencia cuyo objeto es interpretar el significado de la acción social, así como dar, en su virtud, una explicación del modo en que procede esa acción y de los aspectos que produce. En esta definición se comprende por "acción" aquella conducta humana que su propio agente o agentes entienden como subjetivamente significativa, y en la medida que es lo que es (p. 11).

Es evidente el aporte de Weber a las ciencias sociales, su elocuencia y gran profundidad de análisis lo hace uno de los teóricos más controversiales y estudiados de los últimos tiempos. Independientemente, de todas las críticas que podamos expresar, sobre todo a su planteamiento de los tipos ideales, es innegable la gran prodigalidad de su pensamiento y acción.

Actualmente, siguen habiendo diferentes posiciones, en este trabajo se asumirá la hermenéutica como aquella corriente filosófica que, hundiendo sus raíces en la fenomenología de Husserl y en el vitalismo nietzscheano, surge a mediados del siglo XX y tiene como máximos exponentes al alemán Hans Georg Gadamer (1900-2002), Martin Heidegger (1889-1976), y el francés Paul Ricoeur (1913-2005). Todos ellos adoptan una determinada posición en torno al problema de la verdad y del ser, siendo la primera definida como fruto de una interpretación, y el ser (mundo y hombre) como una gran obra textual inconclusa que se comporta de manera análoga a como lo hace el lenguaje escrito. 
Hans Gadamer, introduce a nivel epistemológico una serie de elementos conducentes al estudio de lo social. Su planteamiento fundamental gira en torno al lenguaje como comprensión social. Como continuador de la tradición filosófica de Hegel y Heidegger recupera lo mejor de ambos para proponer su teoría de interpretación de textos (Aguilar, 1992:127).

Según Gadamer el lenguaje es el medio para alcanzar la comprensión, el cual no lo designa como un método sino como un arte. Esto nos conduce a pensar que las relaciones dialógicas o dialogantes dentro de las sociedades es la única forma de acceder a la compresión e interpretación de ellas. Además, hay una concatenación con el hecho de que se establece un proceso en búsqueda de un acuerdo o consenso. Por tanto, la comprensión para él es concebida como la estructura fundamental de la existencia humana y no como un método de las ciencias humanas como lo hace Weber la diferencia de estas concepciones esta que uno se ubica desde la filosofía y el otro desde la sociología en ambos casos se considera el aspecto subjetivo y significante de la acción humana. En Gadamer está presente al considerar que sólo reconociendo lo que ponemos nosotros de nuestra ideología en la interpretación podemos reconocer la alteridad y enfrentarnos al otro. Esto está relacionado con la tradición ya que todos nuestros preconceptos $\mathrm{u}$ opiniones adquieren historicidad como producto de la tradición que hemos heredado (Aguilar, 1992: 134). Suspender nuestros prejuicios, parte esencial del conocimiento, nos enfrenta con una pretendida objetividad que sesgaría la comprensión de la otredad como objeto/sujeto de investigación, este criterio objetivo de entender la investigación nos cerraría el camino que nos lleva hacia el consenso entre las partes dialogantes, pues no veríamos su propia carga subjetiva.

En la relación dialógica se requiere que el otro haga valer sus puntos de vista y que el antropólogo recolecte ese opinión, pero la conversación establece que el interlocutor sepa preguntar pues es la única forma de comprender e interpretar el proceso, ya que de lo contrario sólo sería un reproducir de las vivencias del otro y la negación de la dialéctica pregunta - respuesta (Aguilar, 1992: 150). Aunque podríamos considerar que la dialéctica en Gadamer es mecanicista - positivista por su relación causa y efecto representada por la dupla pregunta y respuesta es importante por la sencilla razón que se le da al diálogo una perspectiva dinámica.

La teoría gadameriana proporciona elementos vitales a la antropología, precisamente por la relación dialógica con la sociedad, que en líneas generales es el objeto epistémico de esta: la relación -identidad alteridad motor fundamental de antropología contemporánea. Esa participación dialogante del antropólogo con los diferentes complejos societales es lo que provee a esta ciencia del material necesario para sus estudios y conclusiones acerca del ser humano socializado.

En Gadamer el diálogo es el modo de comprender e interpretar, visión muy loable en el quehacer metódico de las ciencias sociales, pero en la sociedad existen otros procesos que no son inteligibles a través del lenguaje por ello se debe ir más allá del mero recurso dialógico de comprensión - interpretación. 
No obstante, la hermenéutica contemporánea más que un movimiento definido es una "atmósfera" general que empapa grandes y variados ámbitos del pensamiento, impregnando a autores muy heterogéneos como Michel Foucault, Jacques Derrida, Jürgen Habermas, Otto Apely Richard Rorty.

Para la antropología se trata de un planteamiento epistémico que está en el corazón de la práctica etnográfica, pues está claro que en el quehacer antropológico la comprensión/interpretación de los contextos culturales es indispensable.

Para que la antropología no se sumerja en un éxtasis snobista, acomodaticio y esquizofrénico, pasa por una revisión seria y un re/planteo riguroso de su epistemología, es decir el rescate hermenéutico como esencia científica. Esta espíteme intenta superar la rigidez y unilateralidad refutando la concepción de objetividad como relación externa a los individuos y se plantea la recuperación del contexto social, sus significados, representaciones y múltiples determinaciones.

Reivindicamos al sujeto y la subjetividad como creadores y constructores de la realidad. Analizamos los datos desde una perspectiva comprensiva/interpretativa atendiendo las múltiples subjetividades presentes en el contexto social para de esta manera integrarnos en su mundo de vida: deseos, expectativas, intereses. Pues la contextualización es clave para comprender la significación de los hechos observados. De esta manera, la hermenéutica se transforma en epísteme de la antropología, ya que interpretando el significado subjetivo de la acción social permite describir tales acciones no sólo como proceso mental, sino como práctica social determinada, es decir, acciones contextualizadas, ya que la realidad social no es, sencillamente, algo sostenido sólo por la fuerza de las interpretaciones de los individuos. Es por ello que la interpretación ocupa un lugar fundamental para la etnografía en el estudio y descripción de los fenómenos culturales.

La Hermenéutica en antropología para Paul Rabinow (1992) es, "la comprensión del yo dando el rodeo por la compresión del otro" (p.26). De acuerdo a esta afirmación podemos decir que la interpretación siempre se hace desde la mismidad para percibir la esencia de la diferencia a través de ese rodeo del cual nos habla Rabinow que no es más que poner a prueba nuestra propia imagen ante una alteridad, es decir, poner a prueba el "yo cultural".

\section{LA OPERACIONALIZACIÓN METÓDICA}

La antropología, al igual que todas las ciencias sociales, se acerca a su objeto de investigación a través de un método que la ayude a comprender el fenómeno social que analizan. En este caso se partió de la etnografía como primer nivel fundamental de la investigación antropológica. Para Marcus y Fischer(2000) “La etnografía es un proceso de investigación en el que el antropólogo realiza un estrecho seguimiento observando, registrando y participando de la vida cotidiana de otra cultura, una experiencia etiquetada como el método de trabajo de campo, y luego escribe informes sobre esa cultura, poniendo énfasis en la descripción detallada" (p. 43). Pero este método debe ser algo más que una mera aprehensión de datos que nos permita 
hacer una descripción exacta y objetiva de una sociedad al mejor estilo del funcionalismo-positivista malinowskiano. Por el contrario, se complementa con teorías y técnicas que el investigador maneja previamente y que, al acercarse como observador a otra cultura, re/ define y actualiza. (Alarcón, 2007). Precisamente, como plantea Auge y Colleyn "El investigador debe cuestionar sin cesar sus propios a priori y colocarse en situación de aprendizaje" (2004: 20).

Es por esto por lo que la etnografía como método va más allá de la mera captación de datos. La etnografía debe ser una perspectiva de investigación con un conjunto de operaciones para ir al campo y para luego construir teóricamente un texto escrito.

En esta perspectiva, el antropólogo lleva a cabo su trabajo de campo en el cual recopila información, realiza distintas formas de observación (participante, militante, omnisciente de interacción, etc.) y establece el diálogo con el otro. Al concretarse el diálogo, traspasamos las barreras de la diferencia y nos situamos al lado de la sociedad objeto de estudio en una relación intersubjetiva. No quiere decir esto que tengamos que asumir la visión y cultura del otro -anhelo de muchos investigadoresdejando atrás la nuestra como un traje que se usa y se tira cuando se desee. (Alarcón, 2007).

Geertz (1996),considera que el papel de los estudios etnográficos debe ser una descripción densa en un proceso de interpretación y re-interpretación que se define en el diálogo con el otro. Stephen Tyler (1998), refiriéndose a la etnografía posmoderna, nos dice que "ella pone en primer término al diálogo y no al monólogo, y enfatiza la naturaleza cooperativa y colaborativa de la situación etnográfica en contraste con la ideología del observador trascendental (p. 301).Por consiguiente, el observador no se encuentra aislado del fenómeno que investiga, sino que forma parte de él. Por ello el trabajo de campo es más que un conjunto de técnicas "Es una situación metodológica y también en sí misma un proceso, una secuencia de acciones, de comportamientos y de acontecimientos, no todos controlados por el investigador..." (Velazco y Díaz de Rada, 2006:18).

Por tanto, para el etnógrafo su material primordial es la data empírica y su forma de analizarla es la interpretación y los resultados de una investigación responden a lo "que el etnógrafo seleccionó de lo que entendió, de lo que sus informantes le dijeron, de lo que ellos entendieron" (Sperber, 1991:115).

Si esto es así, el estilo del etnógrafo se centra en lo que Sperber (1991) llama discurso indirecto. En tanto está determinado por el pronunciamiento original del informante, no es una paráfrasis sino más bien un resumen. Esta dinámica conduce a un claro relativismo discursivo, ya que cada etnógrafo condensaría o ampliaría, de acuerdo a su interpretación, lo que observó o lo que el informante dijo.

Como lo dice Sperber (1991) “Por supuesto, los etnógrafos no pueden solamente citar y describir. En la mayoría de los casos deben interpretar, esto es, añadir a las variadas versiones nativas que constituyen una representación cultural, una atípica o 
lo que es lo mismo, una exageradamente atípica, una versión exógena, una versión, por lo tanto distorsionada pero inteligible y relevante para sus lectores" (p. 126).

De acuerdo con esto para Sperber (1991) sería más justo si el etnógrafo se replanteara un giro lingüístico y en vez de decir que trabaja con la interpretación de un hecho, decir que va a interpretar la representación de ese hecho. Sperber (1991) nos dice que el etnógrafo se hace en sus trabajos de campo, a pesar que se nutre de técnicas y experiencias anteriores, cada comunidad estudiada implica un reto para desarrollar técnicas que sean capaces de interpretar las representaciones involucradas, para ello el trabajo de la compresión intuitiva es vital.

El reporte del trabajo de campo está impregnado de conjeturas, de inferencias de una variedad de comportamientos frecuentemente ambivalentes y complejos, inferencias que, si bien la mayoría de las veces son hechas por el etnógrafo, algunas veces son hechas por sus informantes. Así, el más fáctico de los reportes, no logra ser más que un conjunto de enunciados con los cuales el etnógrafo intenta expresar lo que entendió, de lo que sus informantes le dijeron, de lo que ellos entendieron, de lo que pareció relevante al etnógrafo.

La justificación del uso de la interpretación en la Etnografía, descansa, por un lado, en el hecho de que permiten al etnógrafo transmitir su entendimiento de una cultura, y por otro lado, en el hecho de que cuanto puede alcanzarse por la interpretación, no podría alcanzarse a través de un acercamiento puramente descriptivo.

El mundo externo al investigador es construido sobre las bases de su propia cultura y le llevan en una específica dirección. Las posibilidades reales del etnógrafo de comunicar la vivencia cultural del nativo, se reducen a las posibilidades que se tienen de comunicar las reglas de un juego con el uso exclusivo de las reglas de otro juego. La intransferencia de estas reglas bien ilustra sobre la inconmensurabilidad e incomunicabilidad de las culturas y sus lógicas subyacentes entre sí. El etnógrafo no puede sino conocer a través de las representaciones que su cultura le permite hacer. Con estas figuras el etnógrafo sólo puede aspirar a comunicar o mostrar el sentido que para él tiene su objeto de estudio. Como plantea Marc Auge en referencia a los análisis culturales "El antropólogo no traduce, transpone." (2006: 52).De esta manera, la realidad es transformada en objeto antropológico que se muestra para su discusión y comparación con otros estudios etnográficos.

Luego de la dinámica del trabajo de campo, debemos interpretar los datos obtenidos en ese diálogo con el otro, que a la vez se debe relacionar con la teoría y conocimientos anteriores sobre el tema. De esta manera podemos interpretar textos escritos, hablados, actuados y aun de otros tipos y así comprender los discursos orales de los Pütchipü. En el entendido que un texto puede tener múltiples significados (polisemia) o heteroglosia, la interpretación debe conducirnos a rebasar el sentido superficial para llegar al sentido profundo, inclusive oculto; también de encontrar varios sentidos cuando pareciera haber uno solo. En el acto interpretativo hay un relato (oral en este caso), un autor (wayuu Pütchipü) y un intérprete (investigador). Por ello hay que tomar en cuenta la intención del autor pues el texto le 
pertenece. Pero por otra parte, tenemos que darnos cuenta que los relatos ya no dirán exactamente lo que quiso decir el autor, pues ha rebasado su intencionalidad al encontrarse con la subjetividad del investigador, es decir, con su reflexividad. En esta perspectiva, la tarea del investigador es hacerlo decir algo más, así el texto final comprende el significado del autor y el significado del intérprete; es una relación dialéctica y no una mera decodificación del mensaje. Lo producido por el antropólogo no es espejo pasivo de la realidad sino interpretaciones activas construidas sobre ella. Como plantea Rosana Guber (2001) se debe transitar de la reflexividad del investigadora la de los nativos y viceversa.

Es así como el antropólogo interpreta y describe una cultura o determinados aspectos de ella para lectores que no están familiarizados con ella. Lo que se juega en el texto es la relación entre teoría y campo, mediada por los datos etnográficos.

\section{CONCLUSIÓN}

Reconstituir la antropología desde los lineamientos epistémicos de la hermenéutica revitaliza el método etnográfico y lo pone al servicio de la comprensión/interpretación de los fenómenos culturales. Con este nuevo enfoque la antropología se sustrae de los ámbitos empirista del positivismo para lograr una configuración de los contextos culturales que atienda la subjetividad, el cambio y las dinámicas multilocales que se presentan en ellas. Esta redefinición nos distancia de las descripciones de la antropología tradicional trascendental, al situarnos en una perspectiva, que toma encuentra, tanto lo subjetivo como las prácticas sociales de las comunidades que investigamos.

\section{REFERENCIAS}

Aguilar Rivero, Mariflor.1992. Confrontación, crítica y hermenéutica. Gadamer, Ricoeur, Habermas. México. FONTAMARA

Alarcón, Johnny. 2007. Las relaciones de poder político en el pueblo wayuu. Mérida. Ediciones del Vicerrectorado académico-LUZ

Auge Marc y Jean Colleyn: 2004. Qué es la antropología. España. Paidós.

Auge Marc. 2006. El oficio del antropólogo. España. Gedisa.

Geertz, Clifford. 1996. La interpretación de las culturas. Barcelona, Gedisa.

Guber, Rosana.2001. La etnografía. Método, campo y reflexividad. Norma Colombia.

Gutiérrez, Gabriel. 1986. La hermenéutica como método. En: http:/ / visionfilosofica.blogspot.com/2008/07/la-hermenutica-como-mtodogutirrez.html.Consultadoel 13 -03-2015

Marcus, George y Michael, Fischer. 2000. La antropología como crítica cultural. Amorrortu Editores. Argentina.

Mardones, JM.1991. Filosofía de las ciencias humanas y sociales. España. Anthropos.

Martínez, Miguel. 2004. Ciencia y arte en la metodología cualitativa. México. Trillas. 
Rabinow, Paul. 1992. Reflexiones sobre un trabajo de campo en Marruecos. Madrid, JÚCAR

Sperber, Dan. 1991. Etnografía interpretativa y antropología teórica. En: Alteridades. Año1 No 1. México. Universidad Autónoma Metropolitana.

Tyler, Stephen. 1998. La etnografía posmoderna: de documento de lo oculto a documento oculto. EN: El surgimiento de la antropología posmoderna. España, GEDISA Velasco, Honorio y Ángel Díaz de Rada. 2006. La lógica de la investigación etnográfica. San Cristóbal, Editorial Trota.

Weber, Max. 1984. La acción social: Ensayos metodológicos. Barcelona. Península. Weber, Max. 2006. Ensayos sobre metodología sociológica. Buenos aires. Amorrortu Editores. 